\title{
Dietary flavor acceptance in infant rats established by association with effects of nutrient composition*
}

\author{
D. A. BOOTH, ROBERT STOLOFF, and JOY NICHOLLS \\ Department of Psychology, University of Birmingham, P. O. Box 363, Birmingham B15 2TT, England
}

\begin{abstract}
Rat pups come to accept a starch-containing diet and to prefer its flavor or other sensory properties, even when they also have access to the laboratory chow on which their mother is maintained. Protein induces acceptance somewhat more weakly, triglyceride not at all under the conditions used. Nutrient-free material becomes aversive. It is concluded that at least part of the acceptability of a food is an augmentation of its power to elicit ingestatory reactions which has been established by the contingency of physiological effects of ingested carbohydrate or protein on experiences of that foodstuff's distinctive sensory qualities.
\end{abstract}

Hunger is not simply distress or excitement arising from caloric deprivation. Hunger is a motivational state in which food procurement and ingestion itself are directed by an enormous range of relatively arbitrary sensory qualities appertaining to nutritious materials. It has been found recently that a taste or an odor becomes more attractive to the adult rat when the oral stimulus has associated with postingestive effects of a complete diet (Holman, 1969), of carbohydrate (Booth, Lovett, \& McSherry, 1972), or of protein amino acids (Booth \& Simson, 1971; Simson \& Booth, 1973). Such conditioned preferences could contribute to the palatability of normal foodstuffs. Also, in adult rats, very low dietary carbohydrate concentrations (Booth, $1972 \mathrm{~b}$ ) or low energy content of a meal taken after food deprivation (Booth, 1972a) appear to establish conditioned aversions.

In the present study, oral sensory selection and rejection according to previous pairing with a dietary constituent were sought in the infant rat.

\section{EXPERIMENT I}

To assess the importance of prior acquaintance as against several other factors which could influence the development of acceptance of solid foods, infant rats were given a choice between a complete diet of unfamiliar odor, taste, and texture, never presented to the mother, and the maintenance chow which was available to the mother and to the pups in the home cage.

\section{Method \\ Maintenance. Cross-bred Sprague-Dawley-derived albino rats were used in this and all other experiments. A 12-h/12-h dim-bright lighting cycle was applied. Water was presented to all animals at all times through unvalved drinking spouts close to}

*Most of this work was carried out in 1971-1972 at the University of Sussex with support from a grant by the Medical Research Council, U.K. the floor of the cage. A mother (from a week before birth was due) and her litter (until weaning) were housed in a cage with abundant bedding. In this experiment, pellets of maintenance chow (41B diet) were freely available from a hopper covering the lower front of the cage. Fragments tended to accumulate under the bedding within the cage. Each litter was reduced to four males and four females on the day after birth. The young rats were weaned by being removed from the mother and placed in groups of three or four in fresh cages with chow and water ad lib.

Test Diets. The basis of the unfamiliar diets was the unflavored version of Vivasorb (marketed in USA as Vivonex), a nutritionally complete dry diet based on isolated chemicals. It is a white powder readily dissolving in saliva and given a mildly meaty and sweet odor and taste by its maltodextrin, amino acid, and mineral constituents. The $41 \mathrm{~B}$ maintenance diet was used in choice tests in its powder form, a fine flour wetted but not dissolved by saliva and having a fish odor but little taste. In pilot experiments, both powders were badly spilt from open beakers by adult rats; furthermore, unmodified Vivasorb (VIV) was taken in very small quantities by weanlings. Each basic diet was, therefore, given a crumbly paste texture and greater acceptability to the rat by incorporation of petroleum jelly, Vaseline, to $35 \%$ by weight (CHOW-VAS and VIV-VAS). The fourth diet in the choice tests (CHOW-SACC-VAS) was the 41B-Vaseline mixture with $0.1 \%$ sodium saccharin incorporated.

Test Procedures. For intake testing, each pup was isolated in a separate cage in the same room as the home cage. All pups were presented, each day from 14 days after birth, with weighed pellets of maintenance chow for $1 \mathrm{~h}$ after $30 \mathrm{~min}$ of deprivation in the dark or for $2 \mathrm{~h}$ after $1 \mathrm{~h}$ of deprivation in the light. The residuum of pellets in the cage and spillage caught on paper below the cage was weighed. When, in addition, four-way choice tests were made, the pups were isolated and deprived for the last $1 / 2 \mathrm{~h}$ of the bright period $(1230-1300 \mathrm{~h}$ in this experiment) and presented with the diets for the first hour of dark. Each diet had been placed in a $10-\mathrm{ml}$ beaker, weighed to $0.1 \mathrm{~g}$ on a toploading balance, and inserted in a hole sloping at $45 \mathrm{deg}$ drilled at the base of one side of a wooden cube affixed to a spill-tray divided into four. Each beaker was again weighed after the test, together with any spillage in its quarter-tray.

One group of two litters (early choice) was given choice testing from 16 days after birth, sufficiently early to precede any appreciable intake in chow pellet tests. This group was not weaned during the experiment. Another group (late choice), also two litters, was weaned at 21 days after birth (the usual time) and given four-way choices from the following day.

The early choice group at 20 days from birth and both groups 


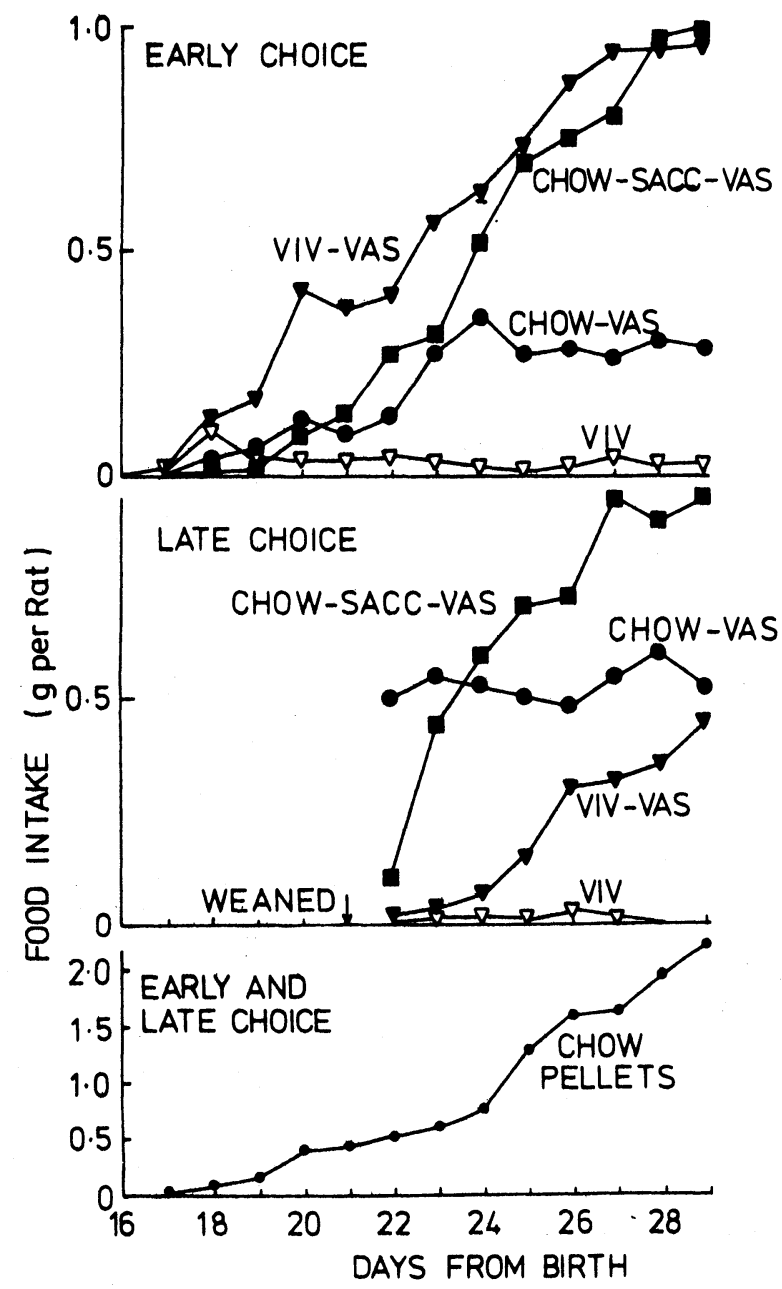

Fig. 1. Food intakes in tests on four experimental diets and tests on pellets of maintenance diet. The four-way choices began at Day 16 in the early choice group and on Day 22 in the late choice group which was weaned. VIV $=$ Vivasorb, a complete synthetic diet. VAS = Vaseline, petrolatum emollient.

at 34 days from birth were given a series of two-way choices towards the middle of the dark period. In a succession of $30-\mathrm{min}$ deprivations and 1-h tests, a choice was given between VIV-VAS and either CHOW-VAS, CHOW-SACC-VAS, or chow pellets or between CHOW-VAS and CHOW-SACC-VAS, the groups being internally counterbalanced with respect to sequences of choices.

\section{Results}

The first detectable intake of solid diet in the test situation occurred on Day 17 from birth (Fig. 1). Readily measurable intakes of chow pellets-the mother's diet in the home cage-developed from Day 18, but so also did intakes of the unfamiliar complete synthetic diet, in the forms both with and without petrolatum (VIV-VAS and VIV, early choice group). Indeed, the intake of VIV-VAS remained above the intake of the mixture of petrolatum and the flour form of maintenance chow (CHOW-VAS) in all subsequent tests, although intake of the grease-free VIV promptly relapsed to very low levels. A preference for saccharin in the greasy chow became definite at Day 24, although it may have been present as early as Day 21 .

The late choice group was weaned on Day 21 from birth and given the choice of test diets for the first time on Day 22. Under these conditions, the first of the four diets to be taken in substantial quantity was CHOW-VAS. This was the test diet which had most in common in orosensory qualities with the solid diet of chow pellets and fragments thereof available in the home cage. Nevertheless, intake of VIV-VAS rose over several days of testing, to rival that of CHOW-VAS. It should also be noted that the incorporation of saccharin did not induce a preference in the very first tests; however, a marked preference for CHOW-SACC-VAS rapidly appeared. The total intake of maintenance chow in the tests was obviously much greater than that of the complete synthetic diet.

The pattern of choices between pairs of diets later on Days 20 and 34 (Table 1) was similar to that seen in the four-way tests. The early choice group on Day 20 preferred VIV-VAS to chow in every form tested. On Day 34, the intake of VIV-VAS was still comparable with that of chow. Indeed, it may require the preference for saccharin, evident at that stage but not on Day 20, to bring chow intake up to or over the level of intake of the other complete diet. In the late choice group, acceptance of VIV-VAS was still considerable, but saccharin preference or chow in a familiar form diverted acceptance to the alternative.

\section{Discussion}

The results indicate that a variety of factors can contribute to the infant rat's increasing tendency to take solid diet in the late stages of suckling and after weaning. It is clearly not necessary that a diet be taken by the mother, or be first presented to the pup in the presence of its mother, for that diet to be detectably sampled and indeed to be consumed in the greatest quantity during a cafeteria test in an isolation cage. This is not likely to have been a facilitatory effect of variety, at least by Day 19 in the early choice group, for the acceptance of nonmaintenance diet developed only slowly in the late choice group which was much more experienced with maintenance chow.

The addition of petrolatum to the complete synthetic

Table 1

Intakes in Choices Between Pairs of Diets

\begin{tabular}{|c|c|c|c|}
\hline & \multicolumn{2}{|c|}{ Early Choice } & \multirow{2}{*}{$\frac{\text { Late Choice }}{\text { Day } 34}$} \\
\hline & Day 20 & Day 34 & \\
\hline VIV-VAS & $0.41^{*}$ & 1.28 & 1.20 \\
\hline CHOW-VAS & 0.11 & 1.03 & 1.36 \\
\hline $\begin{array}{l}\text { VIV-VAS } \\
\text { CHOW-SACC-VAS }\end{array}$ & $\begin{array}{l}0.39 * \\
0.14\end{array}$ & $\begin{array}{l}1.23 \\
1.33\end{array}$ & $\begin{array}{l}0.30 \dagger \\
2.03\end{array}$ \\
\hline $\begin{array}{l}\text { CHOW-VAS } \\
\text { CHOW-SACC-VAS }\end{array}$ & $\begin{array}{l}0.26 \\
0.34\end{array}$ & $\begin{array}{l}0.59 * \\
1.40\end{array}$ & $\begin{array}{l}0.40 \dagger \\
2.11\end{array}$ \\
\hline $\begin{array}{l}\text { VIV-VAS } \\
\text { CHOW PELLETS }\end{array}$ & $\begin{array}{l}0.36^{*} \\
0.19\end{array}$ & $\begin{array}{l}1.17 \\
0.89\end{array}$ & $\begin{array}{l}0.61^{*} \\
1.97\end{array}$ \\
\hline
\end{tabular}

${ }^{*} p<.05 \quad t_{p}<.02$ by two-tailed correlated $t$ tests. 
diet had a very marked facilitatory effect. The initial stages of solid intake were the exception; at that age, motor activity may still be very poorly coordinated by sensory input. The greasiness of a diet improves its acceptability to adult rats, to some extent independently of nutritive value of the oily compound used (Hamilton, 1964). The effect may be innate but not directly sensory, in the sense that a greasy texture may not be preferred for itself but for its easing of the demands on salivation and mastication made by a dry powder.

The facilitatory effect of saccharin presumably is directly sensory. Nevertheless, it showed a marked increase with repeated exposure. The same effect is seen in naive adult rats and in rat pups, in results which support the notion that sweetness is an innate incentive (Jacobs, 1964). The basis for this initial increase in saccharin preference is obscure. Associative effects could contribute in conditions such as those in the present experiment, where food is ingested close in time to the taste of saccharin. Acquisition (Holman, 1969; Booth et al, 1972) and extinction (Le Magnen, 1954) of this factor has been seen in adult rats, sweetness possibly being a privileged stimulus in this respect. A nonassociative factor may also become more evident as oral motor control matures. It also may be subject to initial attenuation by stimulus novelty.

There remains the problem of accounting for the marked preference for the complete synthetic diet over the maintenance chow when each was presented with petrolatum from the start of solids ingestion, and indeed for the rise in VIV-VAS acceptance to approach CHOW-VAS intake, even when choice was delayed until after weaning. Generalization from incentive or reinforcing properties of petrolatum acquired by pairing with chow or saccharin could not by itself explain why VIV-VAS had the greatest intake. Comparisons with intakes of VIV and CHOW-SACC-VAS do not support the idea that unlearned sensory factors play a major role. The remaining possibility is a facilitation of the acceptance of VIV-VAS by its flavor or texture being consistently paired with postingestional appetitive consequences. In the remaining experiments, arbitrary sensory cues were incorporated in the diets and tested for acquired acceptability, to see whether formal evidence for the existence of such conditioning could be obtained in the infant rat.

\section{EXPERIMENT II}

In this experiment, to simplify the paradigm and to vary the conditions of any learning that might occur, diets of differing composition were given to different groups of pups.

\footnotetext{
Method

Procedure. Unweaned pups were maintained in three unreduced litters of 12 each, cross-fostered on the day after birth. Each pup was marked and removed daily for $4 \mathrm{~h}$ into a cage with access to water and, for the middle 2 -h period, to test
}

diet(s). Spills were collected and intakes measured to the nearest $0.1 \mathrm{~g}$. The mother was freely watered and fed on Small Animals Diet pellets throughout the experiment.

Each of four groups of pups experienced only one diet in the tests from Days 20-22 after birth. Powdered maintenance chow was presented as test diet on the following 2 days. Finally, two-sample preference tests were carried out over 3 days, in sequences counterbalanced within groups. Preference for a flavor was determined by presenting a choice between a flavored sample of a mixture of all four diets in equal proportion by weight and an unflavored sample of the same mixture. Preference for the constituents of a training diet.without added flavor was assessed by testing it against the unflavored mixture.

Training Diets and Added Flavors. The diets were pastes of similar consistency composed of nonnutritive bulk alone, or bulk with carbohydrate, protein, or triglyceride added. Constituents were mixed in the following proportions by weight: pure bulk-heavy kaolin 16 , cellulose powder 4 , liquid paraffin 13 ; starch-dextrin 20, cellulose 2, paraffin 11; protein-calcium caseinate 3 , cellulose 4 , paraffin 13 ; fat-maize oil 10 , cellulose 4 , kaolin 16.

Each diet was given a different distinctive taste and smell, the paring of flavor and nutrient being varied between pups so that each pairing was equally represented. The flavor combinations were $0.2 \%$ citric acid plus citral, $0.5 \% \mathrm{NaCl}$ plus geraniol, $0.04 \%$ sodium saccharin plus benzyl acetate, and $0.005 \%$ quinine sulfate plus benzaldehyde. The odorants were added at a concentration of 5 microliters $/ 100 \mathrm{~g}$ of diet.

\section{Results}

As shown in Fig. 2, intakes differed among groups on the third single-diet presentation: the intakes of the group on pure bulk were lower than the intakes of the group on starch diet ( $p<.05$ by Mann-Whitney $U$ test). The intakes of each of these two groups also changed reliably from the first to the third presentation (ps $<.01$ by Wilcoxon tests). Intake of chow powder on the fourth day was similar to the intake of starch diet on the previous day. Subsequent preference tests showed that the flavored bulk diet was rejected $(p<.05)$, and that there may have been a slight preference for the flavored starch diet relative to the unflavored mixture (Fig. 2). However, the flavors themselves, presented in a mixed diet, had not become preferred or rejected relative to the unflavored mixture. This suggested that some other sensory quality such as tex ture was controlling selection. Chow was preferred to all four diets (ps $<.1$ to .01 ).

\section{EXPERIMENT III}

The diets and the isolation and deprivation schedule in this experiment were the same as in Experiment II. Preference tests on added flavor combinations and on dietary constituents were, however, carried out before the series of presentations of flavored single-nutrient diets, as well as after. Also, to vary the conditions of any learning and, indeed, to make it more likely that the added flavors would become used as predictors of the postingestional effects of the diets, each rat was presented with pure bulk and one of the single-nutrient diets on alternate days. 


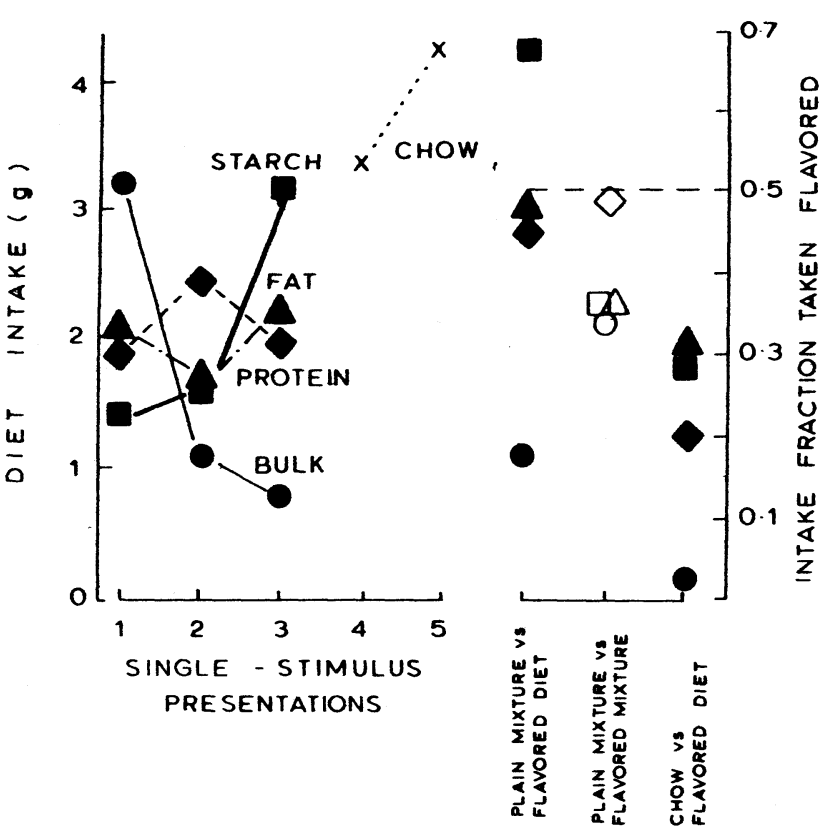

Fig. 2. Intakes of rat pups individually conditioned by repeated presentation of a single diet-pure bulk (circles), starch (squares), protein (diamonds), or triglyceride (triangles). Filled symbols represent flavored single-nutrient diet intakes when it was the only sample present, or when an unflavored mixture or chow was also present (preferences, plotted as fraction of total intake). Open symbols represent the flavor paired with a particular nutrient, but presented in a nutrient mix ture.

\section{Method}

Two litters of 12 pups each were tested from Day 18 after birth. Acceptance tests for flavors and for nutrients were carried out by a two-sample procedure on the first 2 days and by a one-sample procedure the following 2 days. A flavor acceptance was tested by presenting a mixture of starch, protein, and triglyceride diets in equal proportions by weight with addition of the taste and odor combination in question. A nutrient acceptance was tested by presenting the single-nutrient diet without added flavor. In the two-sample tests, unflavored mixture was the alternative. On the fifth day, a sample of powdered maintenance diet was presented. 1

Differential conditioning then ensued for 14 days: flavored bulk diet was presented alternately with flavored single-nutrient diet (either starch, protein, or triglyceride-eight pups in each group). Half of each group had nutrient on the first day of training, half had bulk.

Finally, single-stimulus and then double-stimulus tests of flavor acceptance and of nutrient acceptance were repeated, followed by a test on powdered chow.

All diets were presented in 4-in. metal lids placed on the mesh floor of the isolation cage. Spills were collected on paper towels. Total intakes were measured to the nearest $0.1 \mathrm{~g}$.

\section{Results}

The group of rats alternated between bulk and starch developed a very low intake of the pure bulk diet and a high intake of the starch-containing diet (Fig. 3). The group given the protein-containing diet also tended to take more of that than of the bulk diet, but the difference was more variable and less extreme. No difference between bulk and fat intakes developed in the third group. This contrasts with the differentiation between bulk and fat intakes seen by Hamilton (1964) in adult rats given uninterrupted access to a choice of such diets, a condition not tested in the present work on pups.

In subsequent tests for acquired sensory control of intake, unflavored single-nutrient diets were taken in somewhat greater amounts than unflavored bulk diet on presentation of one sample at a time-a reliable difference in the case of protein-containing diet $(p<.02)$. No such differences had been apparent in the tests prior to conditioning (Fig. 3). This indication of acquired control by texture or some other nutrient-specific oral cue was strengthened by the observation in two-sample tests that an unflavored mixture of the diets was preferred to unflavored simple diets (ps $<.05)$ in all cases except the starch diet.

There was also acquisition of some control of intake

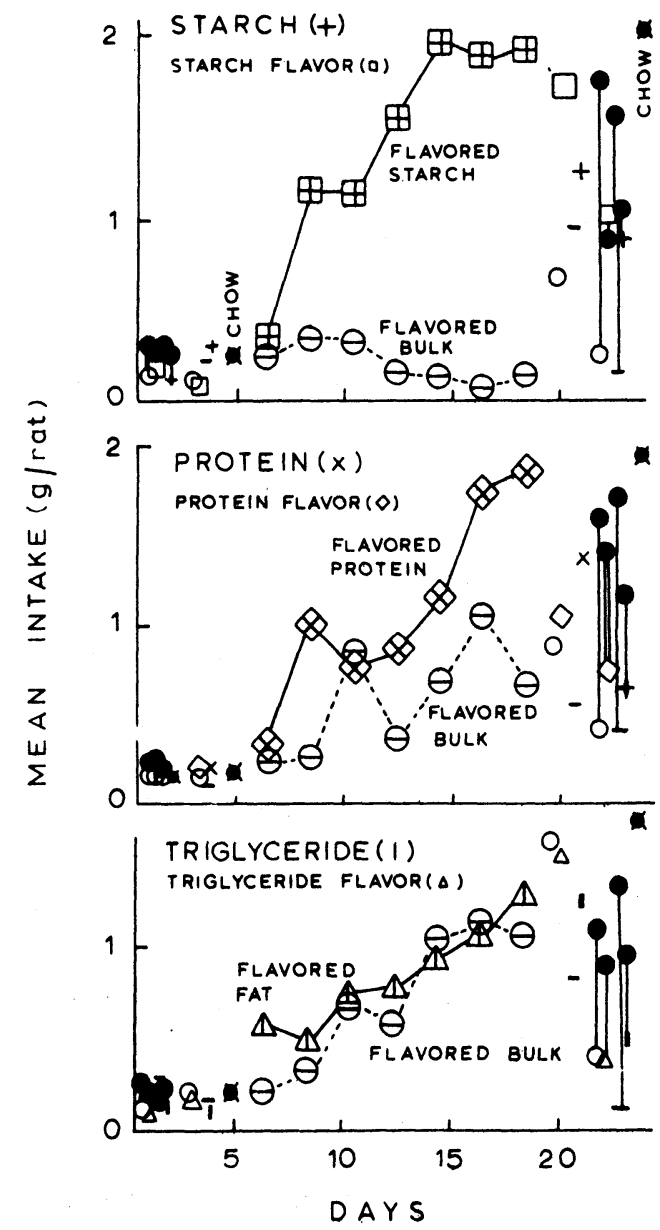

Fig. 3. Intakes of rat pups individually conditioned by alternation between bulk and a single nutrient, differently flavored. Flavors are represented by the open shapes (e.g., bulk-paired flavor is always a circle, empty when presented in a nutrient mix ture rather than in pure bulk). Nutrient composition is indicated by a symbol (bulk is a dash). A filled circle represents a mixture of all nutrients without added flavor. One sample only was presented at a time, except where pairs of data points are connected by vertical lines (two-sample choice tests). 
by flavor. Greater amounts of starch-paired flavor than of bulk-paired flavor were taken in single-sample tests at the end of the experiment (Fig. 3). Also, in two-sample tests, the flavored mixture was rejected in favor of the unflavored mix ture in all cases except the starch-paired flavor. Thus, starch increased the intake of diet having a flavor which had been paired with its presence, and prevented the development of an aversion to either flavor or texture which developed under these conditions in the presence of protein or fat or in the absence of any nutrient. Protein may also have had a reinforcing effect, although a more limited one.

Rats of a similar age were deprived of chow for $1 \mathrm{~h}$, given $1 \mathrm{~g}$ of one of the four diets (unflavored), and then given chow immediately after the diet sample had been consumed. Intakes of chow, relative to intake with no prefeeding on a diet sample, were suppressed by $1.05 \pm 0.31,0.95 \pm 0.18,1.28 \pm 0.25$, and $1.50 \pm 0.55 \mathrm{~g}$ by bulk, fat, starch, and protein, respectively (mean values \pm S.E.). Thus, the preference-inducing and the short-term satiating effects of the diets were not precisely concomitant.

\section{EXPERIMENT IV}

In all the above experiments, the pups were eating maintenance chow at other times on the days when single-nutrient diets were presented. We wanted to see whether fewer aversions were acquired in the absence of this contrast and whether it could be the carbohydrate and perhaps the protein in the diet which normally facilitates the acceptance of solid diets by inducing preferences as the pups began to sample solids. Therefore, more nearly adequate starch- or protein-rich diets were presented. Also, the chow on which the mother was feeding was available to the pups only in the same environment as the experimental diets.

\section{Method}

Each of six pregnant rats was isolated in a mesh-floored cage with access to water through a spout close to the floor and to laboratory chow in a hopper which was out of reach to rat pups either by rearing or by climbing. At 14 days after birth of each litter, a metal sheet barrier was placed in the cage, with holes through which pups could pass but not the mother, to reach diets in 10-ml beakers arranged on the sides of the blocks with spillage trays used in Experiment I.

Three special diets were presented, designated starch-rich, milk-like (protein and fat rich), and bulk-rich. The starch-rich diet was $65 \mathrm{~g}$ cornflower, $10 \mathrm{~g}$ maltodex trin (MD05, Manbré Sugars, London), $5 \mathrm{~g}$ casein, and $10 \mathrm{~g}$ corn oil. The macronutrient composition of the milk-like diet was modeled after rat milk, which is very low in lactose content compared with cow milk or human milk (Brody, 1945): $30 \mathrm{~g}$ corn oil, $25 \mathrm{~g}$ casein, $5 \mathrm{~g}$ maltodex trin, $35 \mathrm{~g}$ kaolin. The bulk-rich diet was $50 \mathrm{~g}$ kaolin, $30 \mathrm{~g}$ paraffin, and $15 \mathrm{~g}$ corn oil. An additional $5 \mathrm{~g}$ in all diets was composed of $4.3 \mathrm{~g}$ of the salt mix of Rogers \& Harper (1965), $0.5 \mathrm{~g}$ of their vitamin mix, and $0.2 \mathrm{~g}$ of choline chloride. These diets were odorized by addition of benzaldehyde, benzyl acetate, or a mix ture of citral and geraniol ( 1 microliter/g). Each litter had a different pairing of odor and nutrient. The position of an odorized diet was changed each day. Chow was ground to a floury texture similar to the other diets and was used as a fourth diet, smelling strongly of the fish flour in it. Diet powders remaining, including the occasional spill, were weighed daily. After this group conditioning, pups were tested individually. On Days 24 and 25 after birth, each pup was isolated with water but without any food for $2 \mathrm{~h}$ and then given a 2-h preference test. In one type of test, three samples of a mix ture of the three special diets in equal proportion by weight were presented, each sample having one of the three odors. In another test, these three odorized mixtures were presented alongside a mixture of one part chow powder and two parts triple diet mixture without added odor, to provide a diet with the odor of chow but little of its taste, tex ture, or appearance.

\section{Results}

The earliest detectable solid intake by a litter generally occurred on the 17 th day after birth. On the following day, the nutrient-containing special diets were taken in substantial amounts, and chow powder and bulk diet were taken in somewhat greater amounts (Fig. 4). On Day 19 from birth, starch-rich diet was eaten by the litters in greater amounts than either milk-like or bulk-rich diet. Intake of the starch-rich diet on that day was as large as that of chow powder. On subsequent days, chow powder intake increased faster than intake of starch-rich diet. Nevertheless, intake of the starch-rich diet continued to rise much more rapidly than intake of the calorically denser milk-like diet, while intake of bulk-rich diet practically ceased. In a subsequent triple-choice test given to individual infant rats, starch-paired odor was considerably preferred to the other two odors (Fig. 4). The odor which had been paired with milk-like diet was slightly preferred to bulk-paired odor. In a quadruple choice, the starch-paired odor was almost as preferred as a chow-containing mixture, and once again was much preferred to the other two added odors.

\section{Discussion}

Maturation of both sensory and motor systems (Campbell \& Mabry, 1972) may well be a precondition of the rat pup's acceptance of solid diet. The presence of the mother and the transmission in mother's milk of a component of chow such as its odor (Le Magnen \& Tallon, 1968; Galef \& Clark, 1972) also facilitate acceptance of chow. Nevertheless, the present results show that these last two factors are not essential to the initiation of substantial intakes of solid diet. Furthermore, the issue remains whether the facilitatory effect of stimuli from the mother or her milk is associative or nonassociative. There might merely have been habituation of disruptive reactions to novelty. It is, however, possible that the facilitation arises from learned safety or-possibly identical-acquired sensory appetite by pairing of otherwise neutral stimuli with nutritive effects of milk.

In the present experiment, chow became strongly favored, over even the starch-rich complete diet. It remains to be seen whether this was because the chow was more nearly adequate or balanced nutritionally, was 


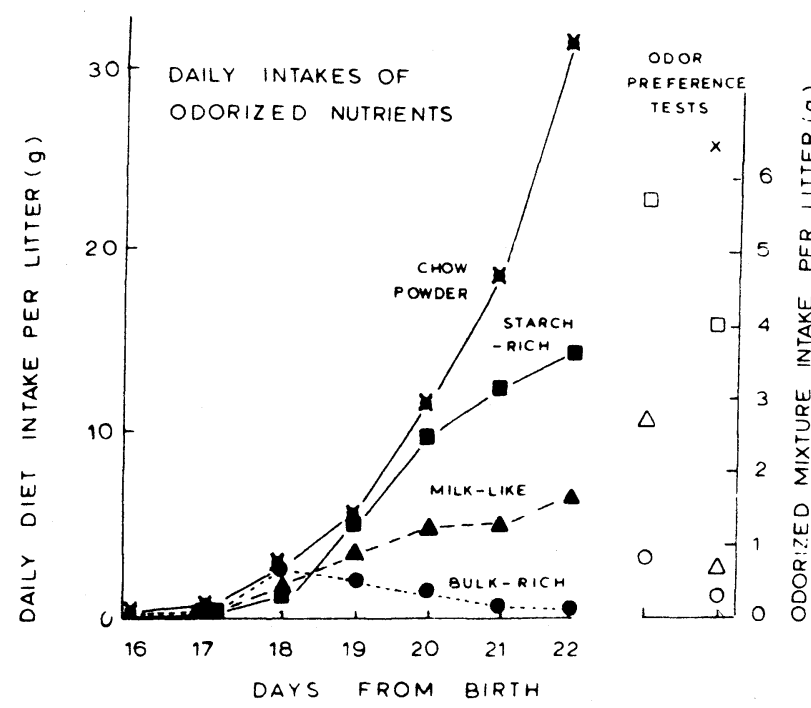

Fig. 4. Intakes during group conditioning and individual testing of pups presented a choice of chow powder (crosses), starch-rich diet (squares), milk-like diet (triangles), and bulk (circles). In the 2 -h odor preference tests, the odors were presented in samples having the same mixed nutrient composition.

substantially more palatable to the maturer rat independently of experience, or was most accepted because of some factor such as prior acquaintance with its odor.

\section{GENERAL DISCUSSION}

Before they began to take detectable amounts of powdered food, the rat pups were observed to suckle, groom, drink from and bite a water spout, nibble and swallow parts of a fecal bolus, and gnaw mesh and the edge of a metal sheet (compare Campbell \& Mabry, 1972). It is, therefore, implausible to regard the sensorimotor organization of feeding as largely a learned act, notwithstanding some vigorous assertions to the contrary (Teitelbaum, 1967). Experiments purporting to show that any motor pattern can be attached to intragastric delivery of nutrient without orosensory mediation have proved to involve an artifact; self-infusion by barpressing appears to be mediated by natural manipulative responding to the acquired incentive value of oral, nasopharyngeal, or conceivably gastric cues predicting parenteral benefits (McGinty et al, 1965; Holman, 1969; Snowdon, 1969; Wasserman, 1973; Rowland \& Nicolaides, 1974). We take the view that carbohydrate (and perhaps protein), or a factor related to its presence in substantial amounts in a balanced diet, produces an internal appetitive US. ${ }^{2}$ That is, temporally related sensory cues become more attractive or better facilitate approach and consumatory movements and secretions. Chemosensory or other oral stimuli may be favored, though not necessarily as stimuli presented by consumption (Domjan, 1973). Hunger and satiety, and the classical effects of lesions and stimulation in the lateral hypothalamic "feeding center," are best understood as changes in oral and perioral sensorimotor facilitation (Brobeck, 1957; Marshall, Turner, \& Teitelbaum, 1971; Turner, 1973; Smith, 1972).

The physiological and behavioral characteristics of the appetitive US remain to be established. Behaviorally, it might involve relief of need-related discomfort, but at an alternative extreme, the US could be nonconscious effects of nutrients (Trémolières, 1972) acting directly in brain "reward" systems (Stein \& Wise, 1971; Booth, 1972c). Postabsorptive effects of ingested carbohydrate (or protein) seem indicated, as in the adult rat (Booth et al, 1972; Simson \& Booth, 1973), because the immediate hunger-reducing effect did not appear to relate to the preference-inducing effect of a nutrient, nor do the likely physiological effects. Nutrient-containing diets and pure bulk provide similar volumes in the stomach immediately following the ingestion of similar weights of diet. Pure bulk and triglyceride would be at opposite extremes of rate of evacuation from the stomach, with the preference-inducing diets (protein and starch) clearing at intermediate rates (Hunt \& Knox, 1968). Osmotic pressures following digestion and eventual caloric yield per unit weight of intake would not correlate with preference-modulating effects either. Speed of utilization or chemospecific effects of glucose and amino acids remain their distinction from fats and bulk. The finding that rat pup intake responds to gastrointestinal filling but not to action of 2-deoxyglucose (Houpt \& Epstein, 1973) may reflect the effects of gastric distension on clearance and absorption rate during test intake, and the lack of connection between glucopoenic feeding and normal appetite control.

In the present experiments, initial acceptance of the test diets was kept as high as possible. The preference-inducing effects were generated by nutrients entering by the normal route. The absence of such conditions may explain the abundance of aversive effects in the previous literature on metabolic or pharmacological modulation of preferences and the relative ineffectiveness of what were intended to be appetitive conditioning procedures (Capretta, Moore, \& Rossiter, 1973; Le Magnen, 1969).

Without weighing against the existence of several other factors entering into the attractiveness of foodstuffs, the present results provide evidence that there exists in the rat an associative mechanism which could operate sufficiently generally to explain much of the consumatory facilitation, search, approach, selection, reward, and pleasure which constitute the desire for food. To the extent that the associative mechanism applies, the so-called primary rewarding properties of food are likely to be a conditioned reinforcement and the instinctive element in hunger may be an associative potential and not a specific drive. Furthermore, such a mechanism provides the missing link between feeding and need, albeit an indirect link and one easily overridden by other factors. 
The mechanism is probably important in other species. Since the present experiments were completed, evidence for similar associative factors in the development of feeding in the infant animal has been presented for the chick by Hogan (1973) and for the guinea pig by Reisbick (1973). When the dog's first experience of meat is in adulthood, it does not salivate to it on that occasion (Bykov, 1957). Bruch (1969) argues that desire for food (which, contrary to both Bruch and Hull, is a motive in behavior, not a physiological state or sensation causing behavior) differentiates in the human child on the basis of consistent association between foodstuffs and their internal effects. Given the strength of the associative effects specifically of carbohydrate, it should be noted that extensive experience with starchy or sugary foods in early life is likely not only to activate any propensity to develop large numbers of adipose tissue cells (Hirsch \& Knittle, 1970) and overefficient conversion of carbohydrate to fat (Yudkin, 1972), but also to encourage an unhealthy preference for these carbohydrate-rich foods in adulthood.

\section{REFERENCES}

Booth, D. A. Conditioned satiety in the rat. Journal of Comparative \& Physiological Psychology, 1972a, 81, 457-471. Booth, D. A. Caloric compensation in rats with continuous or intermittent access to food. Physiology \& Behavior, 1972b, 8, 891-899.

Booth, D. A. Unlearned and learned effects of intrahypothalamic cyclic AMP on feeding. Nature New Biology, 1972c, 237, 222-223.

Booth, D. A., Lovett, D., \& McSherry, G. M. Postingestive modulation of the sweetness preference gradient in the rat. Journal of Comparative \& Physiological Psychology, 1972, 78, 485-512.

Booth, D. A., \& Simson, P. C. Food preferences acquired by association with variations in amino acid nutrition. Quarterly Journal of Experimental Psychology, 1971, 23, 135-145.

Brobeck, J. R. Neural basis of hunger, appetite, and satiety. Gastroenterology, 1957, 32, 169-174.

Brody, S. Bioenerget ics and growth. New York: Reinhold, 1945.

Bruch, H. Hunger and instinct. Journal of Nervous \& Mental Diseases, 1969, 149, 91-114.

Bykov, K. M. Cerebral cortex and the internal organs. New York: Chemical Publishing, 1957.

Campbell, B. A., \& Mabry, P. D. Ontogeny of behavioral arousal: A comparative study. Journal of Comparative \& Physiological Psychology, 1972, 81, 371-379.

Capretta, P. J., Moore, M. J., \& Rossiter, T. R. Establishment and modification of food and taste preferences: Effects of experience. Journal of General Psychology, 1973, 89, 27-46.

Domjan, M. Role of ingestion in odor-toxicosis learning in the rat. Journal of Comparative \& Physiological Psychology $1973,84,507-521$.

Galef, B. G., \& Clark, M. M. Mother's milk and adult presence: Two factors determining initial dietary selection by weanling rats. Journal of Comparative \& Physiological Psychology, 1972, 78, 220-225.

Hamilton, C. L. Rat's preference for high fat diets. Journal of Comparative \& Physiological Psychology, 1964, 58, 459-460.

Hirsch, J., \& Knittle, J. L. The cellularity of obese and non-obese human adipose tissue. Federation Proceedings, 1970, 29, 1516-1521.

Hogan, J. A. Development of food recognition in young chicks: II. Learned associations over long delays. Journal of Comparative \& Physiological Psychology, 1973, 83, 367-373.

Holman, G. L. The intragastric reinforcement effect. Journal of Comparative \& Physiological Psychology, 1969, 69, 432-441.

Houpt, K. A., \& Epstein, A. N. Ontogeny of controls of food intake in the rat: GI fill and glucoprivation. American Journa of Physiology, 1973, 225, 58-66.
Hunt, J. N., \& Knox, M. T. Regulation of gastric emptying. In C. F. Code and W. Heidel (Eds.), Alimentary canal. Vol. 4. Motility. Washington, D.C: American Physiological Society, 1968.

Jacobs, H. L. Observations on the ontogeny of saccharin preference in the neonate rat. Psychonomic Science, 1964, 1, 105-106.

Křreček, J., \& Křrečková, J: The development of the regulation of water metabolism III. The relation between water and milk intake in infant rats. Physiologia Bohemoslovenica, 1957, 6 , 26-33.

Le Magnen, J. Le processus de discrimination par le rat blanc des stimuli sucrés alimentaires et non alimentaires. Journal de Phy siologie, 1954, 46, 414-418.

Le Magnen, J. Peripheral and systemic actions of food in the caloric regulation of intake. Annals of the New York Acad emy of Sciences. 1969. 157, 1126-1156.

Le Magnen, J., \& Tallon, S. Préférence alimentaire de jeune rat induite par l'allaitement maternel. Comptes Rendus des

Marshall, J. F., Turner, B. H., \& Teitelbaum, P. Sensory neglect produced by lateral hypothalamic damage. Science, 1971, 174, 523-525.

McGinty, D., Epstein, A. N., \& Teitelbaum, P. The contribution of oropharyngeal sensations to hypothalamic hyperphagia. Animal Behaviour, 1965, 13, 413-418.

Reisbick, S. H. Development of food preferences in newborn guinea pigs. Journal of Comparative \& Physiological Psychology, 1973, 85, 427-442.

Rogers, Q. R., \& Harper, A. E. Amino acids and maximal growth in the rat. Journal of Nutrition, 1965, 87, 267-273.

Rowland, N. E., \& Nicolaides, S. Periprandial self intravenous drinking in the rat. Journal of Comparative \& Physiological Psychology, in press.

Simson, P. C., \& Booth, D. A. Olfactory conditioning by association with histidine-free or balanced amino acid loads in rats. Quarterly Journal of Experimental Psychology, 1973, 25, 354-359.

Smith, D. A. Increased perioral responsiveness: A possible explanation for the switching of behavior observed during lateral hypothalamic stimulation. Physiology \& Behavior, 1972, 8, 617-621.

Snowdon, C. T. Motivation, regulation, and the control of meal parameters with oral and intragastric feeding. Journal of Comparative \& Physiological Psychology, 1969, 69, 91-100.

Stein, L., \& Wise, C. D. Possible etiology of schizophrenia: Progressive damage to the noradrenergic reward system by 6-h ydrox ydopamine. Science, 1971, 171, 1032-1036.

Teitelbaum, $P$. Motivation and control of food intake. In C. F. Code and W. Heidel (Eds.), Alimentary canal. Vol. 1. Food and water intake. Washington, D.C: American Physiological Society, 1967.

Trémolières, J. A proposed scheme of food behaviour. Bibliographia Nutritio et Dietica, 1972, 17, 144-153.

Turner, B. H. Sensorimotor syndrome produced by lesions of the amygdala and lateral hypothalamus. Journal of Comparative \& Physiological Psychology, 1973, 82, 37-47.

Wasserman, E. Pavlovian conditioning with heat reinforcement produces stimulus-directed pecking in chicks. Science, 1973 $181,875-877$

Yudkin, J. Sugar and disease. Nature, 1972, 239, 197-199.

\section{NOTES}

1. The maintenance diet for Experiments II-IV was pellets of Small Animals Diet (autoclaved version) supplied by Spillers Ltd., London. This diet is, according to the manufacturers, $48 \%$ digestible carbohydrate, $21 \%$ protein, and $4 \%$ crude fat. It is highly palatable and promotes daily body weight increments of $5-6 \mathrm{~g}$ in 200-300 g rats.

2. An unconditioned stimulus is here taken to be an event which changes the S's future involuntary pattern of anticipatory reactions (conditioned response) to whatever sensory input is appropriately related to US onset (conditioned stimulus). The postulation of an US, or reinforcement event, need not depend on an identification of the UR, as some have said; the CR, to be adaptive, may be a response in the same or the opposite direction to the UR, or there may even be no behavioral UR at all, but the existence of the US effect would still have been demonstrated by the elaboration of a CR to any previously ineffective CS of an appropriate modality.

(Received for publication March 26, 1974; accepted April 9, 1974.) 